\title{
The Research on the Relationship between Mongolia's Fertility Rate and Income based the Easterling Model
}

\author{
Xue Jiliang \\ School of Economy and Management, Inner Mongolia University, Huhhot Inner Mongolia 010021, China \\ xuejiliang1981@163.com
}

\begin{abstract}
Along with the economic development and social progress in Inner Mongolia Autonomous Region, and gradually change attitudes, unique ethnic characteristics and the nomadic culture of Mongolia makes its fertility, fertility preferences and livelihood strategy has changed, but the research on the fertility transition did not keep pace with the times. Therefore, this article from the fertility rate measure in 1986-2010, To discover the relationship between the Mongolia fertility rate and income based on Easterling model, found that since 1986, Mongolia's fertility rate and income growth was first inverted $U$ type and then $U$ type ( $N$ type). Mongolia's fertility rate as income growth has growth -- down growth. In the next period, Mongolia people with income growth and fertility policy relaxation, and to continue to grow in the future.
\end{abstract} Mongolia

Index Terms - Fertility Rate, Income, Easterling Model,

\section{Introduction}

Nowadays, Inner Mongolia Autonomous Region is in a significant historical period during which both the economic growth pattern and the social development is on a shift. Along with the economic and social development in Inner Mongolia Autonomous Region as well as gradual shifting fertility concept, the Mongolian's unique national characteristics and nomadic culture have made dramatic changes in its fertility level, fertility preferences and livelihoods strategies. The total fertility rate and that of married childbearing women are 0.94 and 0.99 respectively, both of which are lower than the national average of 1.8. And its evolutional process of fertility rate differing from Han nationality's influences their choice of livelihoods strategies and great changes have taken place in livelihood result, which in turn have a great impact on the sustainable development of economic society. Faced with fast propulsion of the industrialization and urbanization, the Mongolian's unique national characteristics and nomadic culture make dramatic changes in its fertility level, fertility preferences and livelihoods strategies, which provides us with a breakthrough point to study the evolution of the Mongolian population problem in China. Ethnic minority fertility only be fully reflected in the previous census in the country, but more reflects the characteristics of cohort study. For a more direct understanding of ethnic minority fertility, this paper use indirect estimation method to measure the Mongolian fertility, studying the rules of fertility transition, which is of great practical significance and theoretical value.

\section{Literature Review}

Domestic and foreign scholars have carried on in-depth studies on the fertility rate in China from multi-level and multi - angle. Lavely (1984)[1],Yang Tao and Marjorie Mcelroy (2000)analyzed the influence of population policy on fertility behavior from individual changes in fertility behavior after the implementation of the national family planning policy, while(Nancy Birdsall 1983)[2],Chen Wei and ShiMei 2002)[3] analyzed procreation quantity from socio-economic factors such as income, education, urban and rural differences, etc. De la Croix and Doepke(2003)[4]developed a new theoretical link between inequality and growth . In their model, fertility and education decisions are interdependent. The increase in inequality lowers average education and, therefore, growth. We find that this fertility-differential effect accounts for most of the empirical relationship between inequality and growth.

According to questionnaire survey data from Fengcheng Manchu Autonomous County in Liaoning Province, Lu Jiehua and his colleagues [5] analyzed the relationship between the economic income level and the fertility desire of different rural households, finding various factors that restrict and influence the fertility desires of farmers in poor areas today. According to the data obtained from special investigation on fertility desire of fertile women from different economic income families in rural areas of Wenzhou., Huang Lihua and Zhou Chenghong (1995, 1996)[6]studied the influence of income on fertility desire, finding that there are significant differences among fertile women from different economic income families in children quantity demand, gender preference, expectation for children' s success and fertility motivation. Zheng Zhenzhen(2004)[7], Guo Zhigang, Wu Xiwei(2006)[8], Chen Yu, Deng Changrong (2007)[9] and other Chinese scholars looked into the various factors affecting fertility desire systematically from Chinese women's fertility desire.

Chen Zhonghan and Wu Ruijun(2009)[10] proved the phenomenon that people with high income in developed areas have stronger fertility desires by the field research of Minhang District in Shanghai, Using China Health and Nutrition Survey (CHNS) 2006 years of data, Hu Jing [11]conducted an empirical analysis on the relation between the women's income and relative economic status and their fertility desires( the above-mentioned women are married, divorced or widowed female under 52 in China), finding that physical factors played a leading role fertility decision; socio-economic factors determined the number of births of children, and women's income did not have a significant impact on fertility intention. Pan Dan, Ning Manxiu (2010)[12]also found that durable household total income significantly reduce the negative impact on the number of children expected, but 
compared to the income levels of women, the income structure plays a more important role in women's fertility desires.

Into industrialization and urbanization accelerated development period, changes in human capital and fertility has become an important factor affecting agricultural development. Guo Jianxiiong (2005)[13]took human capital, fertility rate and the interactive influence of both as basic variables to observe and analyze the income gap between China's urban and rural areas, finding that the Malthus steady state caused by high fertility rates and low human capital accumulation is the root cause of farmers' difficult income growth. While increasing returns to human capital investment, fertility rate declines in the agricultural sector, so that the human capital can be improved to create a demand for the ability to pay(Guo Jianxiong 2006 ) [14].

At present there are few specific study results on the relation between the ethnic minority fertility and the income. There is no doubt that it is of great significance for research on minority fertility, even for the overall development of the minority population in the future. In this study, Easterling theory is used to investigate and analyze Mongolian fertility rate and its influencing factors and characteristics so that we can grasp the future changes in the overall population, which provides more accurate data reference for the assessment and adjustment of the existing family planning policy and the improvement of the existing old - age security system, making Mongolian population development plan meet the needs of the population and resources, environment, economic and social sustainable development better.

\section{Theories and Methods}

The most classical theory to explain the relationship between the economic income level and the fertility desire is Easterlin relative of population size / relative income hypothesis which is introduced in Fertility Revolution: an Analysis of Supply and Demand written by Richard $\mathrm{A} \cdot$ Easterlin. Richard $\mathrm{A} \cdot$ Easterlin believes that the primary determinants of fertility rate change is the social modernization and culture. In the process of modernization, social and economic variables impact on fertility through a series of "intermediate links", like the demand of children (if the birth control is cheap even free, the number of surviving children expected by parents.), the supply of children (Without any measures to limit the family size or to control birth, the number of surviving children of a couple.) and the cost of birth control (the cost and price paid for birth control and family size limit.

Rather than the entire feedback personal wealth, the core idea of the Easterlin relative of population size / relative income hypothesis contact with the usual economic situation closer. Generally, if people can easily attain an acceptable standard of living just as that of their late childhood, they may marry earlier and have more children. Otherwise, it is possible to delay marriage or at least delay having children.

In fact, Easterlin elaborated a model combining demographic transition and economic transition -- the economic transformation can bring the demographic transition, and demographic transition can also bring economic transformation. This phenomenon often occurred in the early transition period where mortality has decreased and the fertility has not been changed. As the number of young people in the total population increased, average earnings for young people has been decreasing, which makes young people face a tradeoff between family size and material wealth resulting in a decline in the total fertility rate. So far the fertility rate and the family size have been changed. During the transition period of China, using the Easterling model to study the relationship between fertility desire and income of the generation can better explain the action mechanism of economic development on fertility behavior.

Regression model follows the form of simplified benchmark function. According to Grossman and Krueger, the advantages can be listed as follows. Firstly, compared with the estimation of structural equation model, the results of the simplified one can tell the net impact of our income on fertility rate. What's more, With the simplified function, we can avoid the annoyance of collecting the data on the fertility status. The data are difficult to obtain and there is doubt about the effectiveness.

Specifically, we use the following equation as a basic function of the form

$$
F_{t}=\alpha_{0}+\alpha_{1} y_{t}+\alpha_{2} y_{t}^{2}+\alpha_{3} y_{t}^{3}+\varepsilon_{t}
$$

( $\mathrm{F}$ is the fertility index, $\mathrm{y}$ is income)

\section{The Change of Mongolian Fertility Rate}

\section{A. How to measure the fertility rate}

The total fertility rate is an important index to measure the national fertility rate. TOTAL FERTILITY RATE is the sum of the age-specific birth rates (5-year age groups between 10 and 49) for female residents of a specified geographic area (nation, state, county, etc.) during a specified time period (usually a calendar year) multiplied by 5 . This rate estimates the number of children a hypothetical cohort of 1,000 females in the specified population would bear if they all went through their childbearing years experiencing the same age-specific birthrates for a specified time period. It is essential to get the data of annual fertility levels and birth population in studying Mongolia's total fertility rate changes and measuring Mongolia's total fertility rate, However, from the existing data, there is no typically direct retrospective data of the Mongolian history of fertility. There is only the census data of individual points, which can not reflect the changes in process of Mongolian newly-born population and fertility level completely and continuously. It requires reconstructing the historical process of population development basing on the existing data. To this end, this paper adopts the Inner Mongolia autonomous region in 2000 the fifth census in 2010 and the sixth census points race, gender, census data to measure the Mongolian fertility.

Ignoring the case of mortality, to measure the Mongolian fertility level during last 20 years, this article uses childrenwomen ratio and "reverse deposit law" indirectly measuring the general fertility rate and birth rate. Children-women ratio 
refers to the ratio of the number of children and the number of women of childbearing age in the age-specific population data. The ratio reflects the rate of survival children of childbearing women in a certain period. Since it doesn't include the dead children at that time, the children-women ratio can be regarded as effective general fertility rate (CWR). The calculation formula is as follows:

$$
C W R=\frac{\text { 0-4years }(\text { or5-9years }) \text { children }}{15-49 \text { years }(\text { or 20-54years }) \text { women }} \times 1000
$$

Because the survey sixth national census of the Inner Mongolia Autonomous Region is November 1, 2010, so the ratio of the age group of $0-4$ and the number of women of childbearing age, which reflect the fertility level in 2007 November 1 to November 1, 2010 women. The ration of children to women of childbearing is survival measurements, but has certain difficulty to measure all fertility levels. According to the census data, "reverse deposit law" could calculated growth index children.

"reverse deposit law" is based on census age population and death data, to calculate back several years ago age population and birth, to calculate fertility index and general fertility rate, the birth rate. Because of the death of data is not available, the death index does not consider the general fertility rate, birth rate in the use of reverse survival method. Using the census data The Inner Mongolia Autonomous Region fifth census in 2000 and the sixth in 2010, the paper uses "reverse deposit law" to measure the general fertility rate and birth rate in years 1986-2010. The formula for computing:

$$
W_{\mathrm{x}-1}(\mathrm{t}-1)=W_{\mathrm{x}}(\mathrm{t}) / \mathrm{P}_{\mathrm{x}}
$$

In the calculation formula, $W_{x}(t)$ is the number of women at $\mathrm{x}$ years old in $t$ annual. Since ignoring the death, the $P_{x}$ is 1.0. Meanwhile, because age-specific mortality is constant, a set of age-specific according with the actual situation can be selected as a constant. We choose $P_{x}=1.0$, because women agespecific death rate is extremely low, and the impact on measurement results is minimal.

\section{B. The Change of Mongolian Fertility Rate}

From the Figure 1, Mongolian total fertility rate was ascend in first and descend at last. The trend of decline may result from About the Tasks Involved with Population and Family Planning Further Instructions put out in 1982 indicating that for ethnic minorities, it is also necessary to promote birth control, but that could be more flexible. In other words, the birth control plays a decisive role in minority fertility decline. After 1999, the Mongolian fertility rates began to increase. At the same time, it may result from the relaxed regulations compared to Han.

The affecting factors on the changes of the Mongolian fertility in addition to the policy factors, including income level, cultural level, the urbanization level, and women's work nature, and many other aspects. In addition to the policy, there are other factors affecting on the changes of the Mongolian fertility including the income level, cultural level, the level of urbanization and women's labor properties etc. Also, the relationship between income level and fertility rate is considered as normal curve of a center left: fertility rose along with income until earners reached the bracket, at which point it dipped somewhat. It is easy to find that the changes of the Mongolian fertility are caused by the joint action of birth control and social and economic factors. In other words, birth control and economic development codetermine the fertility level. Therefore, this article

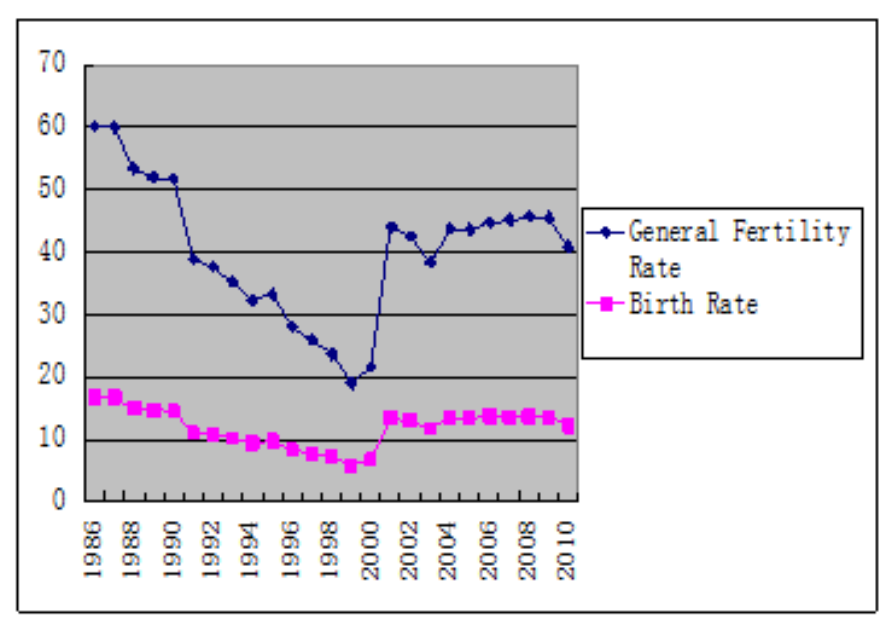

Fig. 1 The Change of Mongolian Fertility Rate(\%o)

\section{The Empirical Results of the Relationship between the Mongolian Fertility and Income}

Given the stability problem of time series data, to avoid serial correlation problems as much as possible, in this article, inducing fertility lag first-order variables, the difference method is used, and the estimation output has been shown in Figure 1 .In the Table1,each model made a joint inspection of income and all $\mathrm{p}$ values are less than $1 \%$, which proved the income level is indeed an important determinant of fertility. For independent variables the general fertility rate and birth rate, Model 2 and Model 4 was significantly better than Model 1 and Model 3; estimation value of Model 2 and Model 4 can be satisfactory for statistical tests. To explain the relationship between energy consumption and economic growth, we select Model 2 and Model 4 in this paper.

Estimation results show that since 1986, the Mongolian fertility rates and income growth with inverted $\mathrm{U}$ before $\mathrm{N}$. With the growth of income, the Mongolian fertility rate showed up-down-up trend. In the next period of time, along with the Mongolian income growth and relaxed fertility policies, the Mongolian fertility rate showed a continued growth trend. It illustrates that the Mongolian fertility in a rapid transition period where the fertility rate change is closely related to income and the income plays a positive role in Mongolian fertility. The parents always consider the marginal rate of childbearing with social status and economic conditions. Usually, the higher the social status and economic conditions of the parents are, the less children 
the parents expected, thus the fertility level decline. From the empirical results and fact, Mongolians did not achieve a higher social status and family income, which means Mongolian fertility may increase in the future.

TABLE I Estimation Output

\begin{tabular}{|c|c|c|c|c|}
\hline \multirow[t]{3}{*}{ variable } & \multicolumn{4}{|c|}{ General Fertility Rate } \\
\hline & Model 1 & & Model 2 & \\
\hline & Parameter & T Test & Parameter & T Test \\
\hline $\mathrm{F}(-1)$ & & & 0.727 & 4.103 \\
\hline Lny & -3.024 & -4.374 & -0.495 & -4.586 \\
\hline \multicolumn{5}{|l|}{$\operatorname{Ln}^{2} y$} \\
\hline $\operatorname{Ln}^{3} y$ & .017 & 4.261 & 0.003 & 4.603 \\
\hline $\mathrm{C}$ & 19.186 & 5.458 & 3.428 & 4.708 \\
\hline $\bar{R}^{2}$ & 0.480 & & 0.689 & \\
\hline $\mathrm{F}$ & 10.142 & & 14.782 & \\
\hline Model type & & & Type N & \\
\hline \multirow[t]{3}{*}{ variable } & \multicolumn{4}{|l|}{ Birth Rate } \\
\hline & Model 3 & & Model 4 & \\
\hline & Parameter & T Test & Parameter & T Test \\
\hline \multicolumn{5}{|l|}{$F(-1)$} \\
\hline Lny & -2.737 & -4.016 & -2.737 & -4.016 \\
\hline \multicolumn{5}{|l|}{$\operatorname{Ln}^{2} \mathrm{y}$} \\
\hline $\operatorname{Ln}^{3} y$ & 0.015 & 3.958 & 0.015 & 3.958 \\
\hline $\mathrm{C}$ & 16.412 & 4.737 & 16.412 & 4.737 \\
\hline $\bar{R}^{2}$ & 0.426 & & 0.683 & \\
\hline $\mathrm{F}$ & 8.175 & & 14.366 & \\
\hline Model type & & & Type N & \\
\hline
\end{tabular}

Since it has prompted growing calls within China for "second child", the study on relationship between income and Mongolian fertility give us the following revelations:

(1) "Second child policy" is on the condition that residents living has been at a high level and a better social security system has been built. Stable low birth level is still the top priority of the current population and family planning work, but at the same time our society faced with the impact of an aging population. So conditional "release the second child" has become the consensus of many domestic experts. This study shows that the key to "Second child policy" is how to Increase the income and improve the social security. The only way to ensure that the "release a second child" will not cause a greater impact on the family, social and personal is to reform and improve infrastructure including income distribution, social security, the household registration system, medical aspects and education, etc.

(2) Do the social economic preparations for fertility rate growth. "Two child" will inevitably impact on the social economy, and will gradually change the structure of labor market, therefore we must continue to deepen reform of factor market, promote the elements of market and improve the labor market segmentation.

\section{Acknowledgment}

This paper was supported by the youth projects of national natural fund: Mongolian fertility change and the mechanism of their livelihood options(71303102).

\section{References}

[1] Lavely, W. R. The Rural Chinese Fertility Transition: A Report from Shifang Xian, Sichuan. Ann Arbor, MI: Population Studies Center, University of Michigan, 1984

[2] Nancy Birdsall, D. T. J. Income and Other Factors Influencing Fertility in China. Population and Development Review, 1983,(4),pp651-675

[3] Chen Wei, Shi Mei. The women's fertility factors in China: empirical analysis of the Easterlin model. Chinese Journal of Population Science. 2002, (2), pp49-53

[4] De la Croix, D. and M. Doepke. Inequality and Growth: Why Differential Fertility Matters?"American Economic Review, 2003 ,93,(4)

[5] Lu Jiehua, Wang Fengmei. The analysis on the economic income level of the farmer and fertility intentions in poor. Northwest Population Journal. 1992, (4), pp38-41

[6] Huang Lihua, Zhou Changhong. The study on the impaction of Rural family economic income for women's reproductive. Journal of Nanjing College for Population Programme Management.1995(2):26-29

[7] Zheng Zhenzhen. Birth intend of women of childbearing age in China. Chinese Journal of Population Science.2004,(5), pp73-78

[8] Guo Zhigang, Wi Xiwei. The application in the study of fertility of Poisson regression in. Chinese Journal of Population Science. 2006, (4), pp2-15

[9] Chen Yu, Deng Changrong. The influence factors of Chinese women's childbearing willing analysis. Chinese Journal of Population Science. 2007, (6), pp75-81

[10]Chen Zhonghan, Wu Ruijun. Impacts of Social and Economic Factors on Environment in Gannan Province. Northwest Population Journal. 2009, (6), pp54-57+61

[11]Hu Jing. Income, Relative Status and Childbearing Willing of Chinese Women. South China Population. 2010, (4),pp3-9

[12]Pan Dan, Ning Manxiu. Income Level, Income Structure and the Childbearing Intention of Chinese Rural Women: An Empirical Study Based on CHNS Data. South China Population. 2010(3):45-50

[13]Guo Jianxiong. Human Capital, the Birth Rate and the Narrowing of the Urban2Rural Income Gap. Social Sciences in China. 2005, (3),pp 27$37+205$

[14]Guo Jianxiong. Human Capital Fertility Rate and Endogenous Agricultural Development: On Impetus to Agricu ltural Development in the Stage of Medium-term Industrialization. Journal of Nanjing University (Philosophy, Humanities and Social Sciences). 2006, (4), pp108-114 